\title{
Hormesis: a fundamental concept in biology
}

\author{
Edward J. Calabrese \\ Department of Public Health, Environmental Health Sciences, Morrill I, N344; Amherst, MA 01003 USA; Tel: +1 413 545 3164; Fax: +1 \\ 413545 4692; E-mail: edwardc@schoolph.umass.edu
}

\begin{abstract}
This paper assesses the hormesis dose response concept, including its historical foundations, frequency, generality, quantitative features, mechanistic basis and biomedical, pharmaceutical and environmental health implications. The hormetic dose response is highly generalizable, being independent of biology model (i.e. common from plants to humans), level of biological organization (i.e. cell, organ and organism), endpoint, inducing agent and mechanism, providing the first general and quantitative description of plasticity. The hormetic dose response describes the limits to which integrative endpoints (e.g. cell proliferation, cell migration, growth patterns, tissue repair, aging processes, complex behaviors such as anxiety, learning, memory, and stress, preconditioning responses, and numerous adaptive responses) can be modulated (i.e., enhanced or diminished) by pharmaceutical, chemical and physical means. Thus, the hormesis concept is a fundamental concept in biology with a wide range of biological implications and biomedical applications.
\end{abstract}

\author{
doi: $10.15698 /$ mic2014.05.145 \\ Received originally: 01.04.2014; \\ Accepted 15.04.2014, \\ Published 23.04.2014.
}

Keywords: hormesis, biphasic, adaptive response, threshold, LNT, hormetic, dose response.

\section{INTRODUCTION}

Hormesis is a biphasic dose response phenomenon, characterized by a low dose stimulation and a high dose inhibition. The term hormesis first entered the scientific lexicon in 1943 by Chester Southam and John Ehrlich [1] based on their observations that extracts from the Red Cedar tree enhanced the metabolism of fungal species at low concentrations. While this was the first use of the term hormesis, from the Greek to excite, there was a substantial history of biphasic dose responses from the 1880 s onward with the first experimental evidence concerning the effects of numerous disinfectants on the metabolism of yeast [2,3]. These findings were confirmed and extended by others with most research using plant or microbiological models. Such information became incorporated into various textbooks in botany [4,5] and microbiology [6-9] during the early to mid portions of the $20^{\text {th }}$ century [See 10-14 for a detailed summarization]. Considerable efforts in the area of plant biology and agriculture assessed how this biphasic dose response concept could enhance crop production with exposures in the low dose range.

\section{HORMESIS: HISTORICAL CONFLICTS}

Despite considerable research documenting the occurrence of biphasic dose responses during these early years, this concept became embroiled in the long dispute between homeopathy and what we call today traditional medicine. Soon after Schulz first observed the occurrence of the biphasic dose response with yeast, he came to be- lieve that he had discovered the explanatory principle of homeopathy [15]. Since Schulz was a traditionally trained physician and professor of pharmacology he came under immediate and intense criticism by his medical colleagues at the University of Greifswald and others in the traditional medicine community and soon became marginalized by his peers. However, what was significant was that Schulz essentially handed homeopathy the biphasic dose response, thereby scooping traditional medicine on the concept of the dose response. In retrospect it is not difficult to understand why traditional medicine and its core disciplines, such as pharmacology, did not accept Schulz's biphasic dose response. It was not possible for traditional medicine to accept the dose response of their intense rival. Doing so would provide credibility to homeopathy. In fact, traditional medicine organized to discredit Schulz and his biphasic dose response model all the while attacking homeopathy, which, at the time, was fairly formidable [16-19]. Eventually traditional medicine would adopt its own dose response model, gravitating to the threshold dose response as a centerpiece in pharmacology, therapeutics and toxicology.

The $20^{\text {th }}$ century would see a rapid reversal in the fortunes of homeopathy, becoming highly marginalized, whereas traditional medicine would become enormously successful [15]. In 1900 there were two dozen homeopathic medical schools in the United States. Some 25 years later there were but three. Mirroring these institutional changes, the biphasic dose response became marginalized, omitted from textbooks, curricula, research funding and regulatory procedures. In essence, it received the equiva- 
lent of a scientific and academic death sentence. By the time of Schulz's death in 1932 his biphasic dose response model had little standing in the medical community, some five decades after his initial discovery. In contrast, the threshold dose response became broadly accepted, incorporated into all pharmacology and toxicology textbooks and became the basic dose response model for government mandated hazard assessments for chemicals, drugs and radiation.

Yet the concept of the biphasic dose response did not die. As noted above, many investigators continued to report such findings. These biologically oriented researchers were not part of the internecine battle between homeopathy and traditional medicine. However, there was little attempt to develop a dose response concept consolidation as most of these investigators, a number of whom had become prominent, would migrate into academic administration or government positions leaving the biphasic dose response behind. Thus, there was little effective leadership on this topic. Given the powerful opposition from traditional medicine and its impact on pharmacology and toxicology, the biphasic dose response failed to thrive as a concept during the middle decades of the 20 century [15].

Amidst the various scattered, unfocused and yet surprisingly numerous publications of biphasic dose response relationships emerged the work of Thomas Luckey, a biochemist working with gnotobiotic experimental systems. In the 1950 s Luckey $[20,21]$ reported that hormetic dose responses occurred with various antibiotic treatments in poultry. Luckey would eventually turn these and other observations into a general biological concept, not unlike that proposed by Schulz some 70 years earlier, reactivating the dose response debate. However, Luckey's synthesis would occur with ionizing radiation hormesis rather than chemical hormesis. Substantial examples of radiation and chemical radiation had been reported in the early literature on hormesis. Yet, researchers in these two domains rarely communicated nor cited each other. Nearly 25 years after his initial observation, Luckey [22] would provide the first detailed book on hormesis, with an exclusive focus on radiation. This book would stimulate the electric power industries in Japan and the US to organize the first conference on radiation hormesis in August, 1985.

Parallel to the efforts of Luckey during the 1970s and 1980 s were scientists in a variety of disciplines who reported on the occurrence of biphasic dose responses, sometimes using the term hormesis but often with terms such as J- or U-shaped, bimodal, bidirectional, or others. Amongst the leaders was Tony Stebbing, an ecotoxicologist from the UK, who developed a cybernetic regulatory mechanism to account for hormetic dose responses [23]. On the pharmacological side, the work of Szabadi [24] was equally important as biphasic dose responses were reported in numerous receptor systems, with the responses mediated via the interaction of different receptor subtypes via the same agonist. Thus by the 1980s the hormesis concept experienced a multidisciplinary resurgence within small pockets of the biological and biomedical sciences.
While there was the renewal of interest in hormesis within the scientific community, interest also emerged from the regulated chemical and electric industry communities. These groups had recently become targeted for regulation using the conservative linear-no-threshold (LNT) dose response model for carcinogens. While the industry initially challenged the legitimacy of the LNT model it was unsuccessful because its alternative to the LNT, that is, the threshold model, was difficult to differentiate from the LNT model when only a few doses were used in hazard assessment cancer bioassay studies. It became clear that there may be a better chance to challenge the LNT model via the use of a hormetic model since the two types of dose responses were more fundamentally different and gave a greater possibility of being differentiated within experimental systems.

This situation was both good and bad for the hormesis concept. It generated interest from the regulated community but also suspicion and opposition from many in the regulatory agencies, especially in the EPA and public health communities. However, regardless of how regulatory agencies might consider hormesis it is a biological concept and could be studied and assessed.

\section{HORMESIS: A RESURGENCE}

The 1990s saw continued interest in the hormesis concept and in the development of more systematic and rigorous assessment of the historical and contemporary dose response literature. Developments of multiple large data bases of hormetic dose responses using rigorous a priori entry and evaluative criteria revealed that hormesis is very general, being independent of biological model, endpoints measured and chemical class. These assessments revealed that hormesis dose responses could occur following either a direct stimulation or via an overcompensation stimulation following an initial disruption in homeostasis. Analysis of more than ten thousand hormetic dose responses indicated that the magnitude of the hormetic dose response was invariably modest, with the vast majority of hormetic dose responses having a magnitude of response less than two-fold greater than the control group [25]. Most maximum responses were only about $30-60 \%$ greater than the control group. The modest nature of the hormetic dose response is its most distinct feature but also making it more difficult to prove and replicate. Of significance in this regard is that whether the hormetic response occurred via a direct stimulation or via an overcompensation response the quantitative features of the dose responses were similar. This also revealed that it was necessary to study hormesis within a dose-time-response context. Consistent with these observations is a recent report detailing specific mechanisms for 400 hormesis dose responses via various receptors and cell signaling pathways [26]. These findings indicate that hormetic dose responses can occur via a broad range of mechanisms and that the quantitative features of the hormetic dose response is also independent of mechanism. 
The fact that hormetic dose response features were independent of biological model, endpoint, inducing agent and mechanism was a striking and unexpected cumulative observation based on an enormous amount of data. Given the extensive diversity in biological systems how could one account for such a consistent and integrative series of observations? These findings lead to the conclusion that the hormetic stimulation provided the first quantitative description of biological plasticity. It revealed how much "gain" there is in biological systems. This capacity is modest, only about $30-60 \%$. This trait is one that has been highly conserved as it exists from plants to humans and at the multiple levels of biological organization. Furthermore, we found that hormetic dose response could in fact be mathematically framed within an allometric relationship, similar to parameters that are functions of body weight or surface area [27]. Thus, the concept of hormesis and its accounting for the quantitative features of plasticity represents a discovery of a fundamental biological concept. It is also important to note that this basic concept escaped detection by the scientific community for many decades, perhaps another unknown victim of the battle between homeopathy and traditional medicine.

Detailed examinations of major biomedical content areas such as neurosciences [28], immunology [29], tumor cell biology [30], wound healing [31], aging/biogerontology [32], plant biology [33], and others revealed that hormetic dose responses were common. Entire areas of the pharmaceutical industry have been built upon the hormetic dose response including anxiolytic drugs, anti-seizure drugs, memory enhancing drugs and other areas [28]. The expanding areas of pre-conditioning and post-conditioning are also founded upon the hormetic dose-response [34].

The hormetic dose response, which is a measure of biological performance for integrated endpoints, imposes a limitation on the magnitude of the induced biological response, thereby affecting the potential for a pharmaceutical induced benefit. This concept has implications for pharmaceutical companies for drug discovery and development as well as how to optimize the design of clinical trials.

Further investigations have assessed the frequency of the hormetic dose response in the biomedical and toxicological literature. Using rigorous a priori and evaluative criteria an assessment over 21,000 articles in three toxicological and life sciences journals over a thirty year period determined that the frequency of the hormetic dose response was $37 \%$ [35]. Follow up studies using additional new very large independent data bases revealed that the hormetic dose response model was far more effective than either the threshold or the linear dose responses model to make accurate predictions in the low dose zone [36].

\section{HORMESIS: IN PERSPECTIVE}

This summary reveals that the biomedical, toxicology and regulatory communities made a fundamental error on the nature of the dose response in the low dose zone during the early decades of the 20th century. This mistake was the result of multiple factors, including the conflict between homeopathy and traditional medicine as well as the fact that assessing hormetic dose responses is difficult, requiring more doses than normal, greater sample sizes, and a time component along with the heightened need to replicate such findings. During most of the 20th century insights into the generalizability or the quantitative features of the hormetic dose response were not developed. Thus, there were many reasons for the failure of the hormetic dose response to thrive and mature. In contrast, the threshold dose response was readily adopted by the medical community and quickly integrated into education, training, regulation and therapeutics. This rapid transition not only reflected the important role that the dose response would play in these areas but also that the scientific and regulatory communities failed to vet the capacity of the threshold model to make accurate predictions in the low dose zone prior to accepting this model as the default standard. Thus, the scientific and regulatory communities gave a free pass to the threshold model, permitting public health standards to be based upon an unvetted model. Of course, some 60 years later the threshold and its companion linear (LNT) model failed extensive validation tests while only the marginalized and historically excluded hormetic model passed.

The hormetic dose response model is still facing challenges for recognition and acceptance. The challenges start with the fact that all regulations have been built upon on a hazard assessment scheme that assumes the existence of a threshold dose response. Only three high doses are typically used in such testing, making it virtually impossible to observe a hormetic dose response. Thus, the rules of the testing game significantly affect the outcome. It is important to recognize that past testing and regulations were based on incorrect dose response assumptions. The incorporation of hormetic concepts into the hazard assessment process also requires more doses, animals and a heightened need for replication, all significant disincentives. Despite these challenges and obstacles there is a dose response revolution and at the center of it is the hormesis dose response. This is reflected in the rapid and extensive growth of its citations in the biomedical community (i.e. from about 10 citations/year in the 1980s to nearly 6000 in 2013 in the Web of Science/Knowledge) and in its incorporation into leading textbooks in toxicology and pharmacology, something absent during the 20th century. The hormesis concept helps researchers better address the issue of low dose responses, including areas such as enhancing adaptive capacities, slowing down the onset of chronic degenerative diseases and improving biological performance in many other ways. Hormesis was selected many millions of years ago and incorporated into the diversity of life. The biomedical community has now started to exploit it for public health, therapeutic and commercial benefit. 


\section{ACKNOWLEDGEMENTS}

Long-term research activities in the area of dose response have been supported by awards from the US Air Force (S11330000000017) and ExxonMobil Foundation (S18200000000156) over a number of years. Sponsors had no involvement in study design, collection, analysis, interpretation, writing and decision to submit.

\section{CONFLICT OF INTEREST}

The authors declare no conflict of interest.

\section{REFERENCES}

1. Southam CM, Ehrlich J (1943). Effects of extracts of western redcedar heartwood on certain wood-decaying fungi in culture. Phytopathology 33:517-524.

2. Schulz H (1887). Zur Lehre von der Arzneiwirdung. Virch Archiv Pathol Anat Phys Klin Med 108:423-445.

3. Schulz H (1888). Uber Hefegifte. Pfluger's Arch Physiol 42:517-541.

4. Duggar BM (1911). Plant physiology, with special reference to plant production. Macmillan, New York.

5. Brenchley WE (1927). Inorganic plant poisons and stimulants, second edition. Cambridge University Press, London.

6. Rahn O (1932). Physiology of Bacteria. P. Blakiston's Son and Co., Philadelphia.

7. Salle AJ (1939). Influence of environment upon bacteria. In: Fundamental principles of bacteriology with laboratory exercises. McGrawHill Book Company, Inc., New York.

8. Clifton CE (1957). Death of bacteria. In: Introduction to bacterial physiology. McGraw-Hill Book Company, Inc. New York.

9. Lamanna C, Mallette MF (1965). Basic bacteriology: Its biological and chemical background. $3^{\text {rd }}$ Edition. The Williams \& Wilkins Co., Baltimore.

10. Calabrese EJ, Baldwin LA (2000). Chemical hormesis: Its historical foundations as a biological hypothesis. Hum Exper Toxicol 19:2-31.

11. Calabrese EJ, Baldwin LA (2000). The marginalization of hormeiss. Hum Exper Toxicol 19:32-40.

12. Calabrese EJ, Baldwin LA (2000). Radiation hormesis: Its historical foundations as a biological hypothesis. Hum Exper Toxicol 19:41-75.

13. Calabrese EJ, Baldwin LA (2000). Radiation hormesis: The demise of a legitimate hypothesis. Hum Exper Toxicol 19:76-84.

14. Calabrese EJ, Baldwin LA (2000). Tales of two similar hypotheses: The rise and fall of chemical and radiation hormesis. Hum Exper Toxicol 19:85-97.

15. Calabrese EJ (2011). Toxicology rewrites its history and rethinks it future: Giving equal focus to both harmful and beneficial effects. Environ Toxicol Chem 30(12):2658-2673.

16. Wels P (1933). The life time work of Hugo Schulz. NaunynSchmied Arch Exper Pathol Pharmakol 170:744-757.

17. Bohme H (1986). Hugo Schulz (8/6/1853-7/13/1932) his life and work. Dissertation, Berlin: Medical Department of the Freien University of Berlin. (Translated by JM Ryan, University of Massachusetts, Amherst).

18. Clark AJ (1933). Mode of action of drugs on cells. Arnold, London.

\section{COPYRIGHT}

(C) 2014 Calabrese. This is an open-access article released under the terms of the Creative Commons Attribution (CC BY) license, which allows the unrestricted use, distribution, and reproduction in any medium, provided the original author and source are acknowledged.

Please cite this article as: Edward J. Calabrese (2014). Hormesis: A Fundamental Concept in Biology. Microbial Cell 1(5): 145-149. doi: 10.15698/mic2014.05.145

19. Clark AJ (1937). Handbook of experimental pharmacology. Verlig Von Julius Springer, Berlin.

20. Luckey TD, Gordon HA, Wagner M, et al. (1956). Growth of germfree birds fed antibiotics. Antibiot \& Chemother 6(1):36-40.

21. Luckey TD (1959). Modes of action of antibiotics in growth stimulation. Recent Progr Microbiol 7:340-370.

22. Luckey TD (1980). Ionizing radiation and hormesis. CRC Press, Boca Raton, FL.

23. Stebbing ARD (1982). Hormesis-the stimulation of growth by low levels of inhibitors. Sci Tot Environ 22:213-234.

24. Szabadi E (1977). Model of 2 functionally antagonistic receptor populations activated by same agonist. J Theor Biol 69:101-112.

25. Calabrese EJ, Blain RB (2011). The hormesis database: The occurrence of hormetic dose responses in the toxicological literature. Reg Toxicol Pharm 61(1):73-81.

26. Calabrese EJ (2013). Hormetic Mechanisms. Crit Rev Toxicol 43(7):580-606.

27. Calabrese EJ (2013). Biphasic dose responses in biology, toxicology and medicine: Accounting for their generalizablity and quantitative features. Environ Poll 182:452-460.

28. Calabrese EJ (2008). Neuroscience and hormesis: Overview and general findings. Crit Rev Toxicol 38:249-252.

29. Calabrese EJ (2005). Hormetic dose-response relationships in immunology: Occurrence, quantitative features of the dose response, mechanistic foundations, and clinical implications. Crit Rev Toxicol 35:89-295.

30. Calabrese EJ (2005). Cancer biology and hormesis: Human tumor cell lines commonly display hormetic (biphasic) dose response. Crit Rev Toxicol 35:463-582.

31. Calabrese EJ (2013). Historical foundations of wound healing and its potential for acceleration: Dose-response considerations. Wound Rep Regen 21(2):180-193.

32. Calabrese EJ (2013). Hormesis: Toxicological foundations and role in aging research. Exper Gerontol 48(1):99-102.

33. Calabrese EJ, Blain RB (2009). Hormesis and plant biology. Environ Poll 157(1):42-48.

34. Calabrese EJ, Bachmann KA, Bailer AJ, Bolger Pm, Borak J, Cai L, Cedergreen N, Cherian MG, Chiueh CC, Clarkson TW et al. (2007) Biological stress response terminology: Integrating the concepts of adaptive response and preconditioning stress within a hormetic doseresponse framework. Toxicol Appl Pharm 222(1):122-128.

35. Calabrese EJ, Baldwin LA (2001). The frequency of U-shaped doseresponses in the toxicological literature. Toxicol Sci 62:330-338. 
36. Calabrese EJ, Stanek EJ, Nascarella M, Hoffmann GR (2008). Hormesis predicts low-dose responses better than threshold models. Inter J Toxicol 27(5):369-378. 